\title{
Analysis of Related Problems of Pelvic Abdominal Adhesion
}

\author{
Zhenzhen Jia, Lihong Zhu* \\ Shaanxi University of Chinese Medicine, Xianyang 713000, Shaanxi Province, China \\ *Corresponding author: Lihong Zhu, show647100@sina.com
}

\begin{abstract}
Pelvic abdominal adhesion is a major problem in obstetrics and gynecology. The occurrence of adhesion complications, the difficulty and risk of adhesion reoperation, and the problem of postoperative adhesion not only cause great trouble to the physical and mental health of patients, but also increase the economic burden for the family and social medical system.
\end{abstract}

Keywords: Adhesion; Cause; Mechanism; Complication; Preventive measures

Publication date: November 2021; Online publication: November 30, 2021

\section{Introduction}

Adhesion refers to the abnormal fibrous structure between normal organs or tissues, which makes the original tissue structure disordered, rigid, or even ectopic. Surgery is the most common cause of adhesion. $63 \% \sim 97 \%$ of patients have adhesion after abdominal surgery, and more than 1/3 of women are hospitalized again because of adhesion diseases after gynecological surgery. Reoperation of pelvic and abdominal adhesions will face many problems, such as the difficulty of surgical site exposure, intraoperative bleeding, separation and injury of surrounding organs, postoperative intestinal obstruction, re adhesion as well as economic burden.

Chengying Xing ${ }^{[1]}$ et al. studied the correlation between the occurrence of pelvic adhesion and Chlamydia trachomatis (CT) and Ureaplasma urealyticum (UU) infection. It was found that pelvic adhesion was related to CT and UU infection. Yuqing Deng et al. proposed that in patients with tubal pregnancy, the incidence of pelvic adhesion in patients with positive Ureaplasma urealyticum UU DNA and CT DNA in cervical secretion was about 70\%. A master's thesis of Guangxi University of Chinese Medicine showed that $16.5 \%$ and $17.8 \%$ of patients with pelvic adhesion had Chlamydia trachomatis and Ureaplasma urealyticum infection]. Barcz, e et al. ${ }^{[2]}$ confirmed that the concentration of interleukin-6 (IL-6) in patients with endometriosis adhesion was significantly higher than that in patients without adhesion. At the same time, it was found that vascular endothelial growth factor A (VEGF-A) was negatively correlated with pelvic adhesion, suggesting that VEGF signal pathway may be a therapeutic target to prevent the formation of peritoneal adhesion. Studies have shown that the time and dose of pelvic radiotherapy are related to the formation of adhesion, and the incidence of pelvic and abdominal tuberculosis is directly proportional to the degree of adhesion. To sum up, the occurrence and development of adhesion is not the result of the influence of a single factor, but a complex process of multiple factors.

$\mathrm{Hu} \mathrm{Q}$ et al. ${ }^{[3]}$ found that human adhesive tissue was significantly thickened and HE staining was full of various cells. The interaction between different types of immune cells may lead to the formation of peritoneal adhesion after trauma, and these different types of cells play different roles in different stages. 
Fischer et al. ${ }^{[4]}$ showed that a large number of membrane bridges between mesothelial surfaces cause adhesion, which means that the pathological changes of mesothelial cells are the main component of early adhesion. Uyama et al. ${ }^{[5]}$ proved that adhesion related myofibroblasts mainly come from mesothelial cells (MC) and produce adhesion. MCs secretes a large amount of VEGF through mesothelium to mesenchymal transition (MMT). Angiogenesis is another important morphological feature of fibrous belt, and the high secretion of VEGF is an important factor leading to vascularization in the process of adhesion formation. Therefore, the potential goal of reducing adhesion formation may be to regulate the MMT program.

Infertility, intestinal obstruction, chronic abdominal pain and pelvic pain are the most common complications of pelvic and abdominal adhesions, which not only puzzles the physical and mental health of patients and increases the economic burden, but also is a huge problem faced by gynecologists and obstetricians. At present, fine surgical techniques and the use of anti-adhesion materials are the main measures to prevent adhesion. Fine surgical technology is the basis for reducing adhesion. It includes fully considering the surgical indications, following the principle of minimally invasive surgery, gently treating the tissue, avoiding damage to adjacent organs, effectively stopping bleeding, avoiding tissue ischemia, foreign body residue, dehydration and infection, using surgical instruments with caution, using unresponsive fine suture materials and auxiliary anti adhesion materials, etc.

Most effective anti-adhesion substances can reduce the formation and re-formation of adhesion, but cannot prevent the occurrence of adhesion. So far, no substances that can prevent adhesion have been found. VEGF signaling pathway may be a therapeutic target to prevent peritoneal adhesion. Regulating MMT process may be a research direction to prevent postoperative adhesion. The existing evidence shows that the use of anti-adhesion materials is reasonable. However, the mechanism of adhesion still needs to be further studied and improved to find a breakthrough point to prevent adhesion and provide safe, simple and effective anti-adhesion materials for clinic. However, good surgical techniques should be put in the primary position of preventing adhesion from beginning to end, and it cannot be replaced by anti-adhesion materials

\section{Disclosure statement}

The authors declare no conflict of interest.

\section{References}

[1] Xing C, Cheng Z, Shi Y, et al., 2005, Relationship between Pelvic Adhesion and Infection with Chlamydia Trachomatis and Ureaplasma Urealyticum. Chinese Journal of Clinical Obstetrics and Gynecology, 6(1): 25-28.

[2] Barcz E, Milewski Ł, Dziunycz P, et al., 2012, Peritoneal Cytokines and Adhesion Formation in Endometriosis: An Inverse Association with Vascular Endothelial Growth Factor Concentration. Fertility and Sterility, 97(6): 1380-1386.e1.

[3] Hu Q, Xia X, Kang X, et al., 2017 A Review of Physiological and Cellular Mechanisms Underlying Fibrotic Postoperative Adhesion. International Journal of Biological Sciences, 17(1): 298-306.

[4] Fischer A, Koopmans T, Ramesh P. et al., 2020, Post-Surgical Adhesions are Triggered by Calcium-Dependent Membrane Bridges between Mesothelial Surfaces. Nature Communications. 11: 3068.

[5] Uyama N, Tsutsui H, Wu S. et al., 2019, Anti-interleukin-6 Receptor Antibody Treatment Ameliorates Postoperative Adhesion Formation. Scientific Reports. 9:17558. 